\section{Dendritic spines shape up}

For some time, the role of the spine neck in dendrites has been controversial. Kasai and colleagues go some way to resolving this debate and propose that the shape of spine necks is crucial for determining dendritic $\mathrm{Ca}^{2+}$ signalling, and therefore longterm potentiation.

The regulation of synaptic plasticity depends on increases in intracellular $\mathrm{Ca}^{2+}\left(\left[\mathrm{Ca}^{2+}\right]_{\mathrm{i}}\right)$, mediated by glutamate receptors that are sensitive to NMDA ( $N$-methyl-D-aspartate). Synaptic function and plasticity are thought to depend on the structure of synapses, and long-term plasticity seems to occur more efficiently in smaller spines. However, the relationship between spine structure, including spine neck structure, and NMDA receptor (NMDAR)dependent $\mathrm{Ca}^{2+}$ signalling has, until now, not been established.

Kasai and colleagues used twophoton excitation to uncage and activate a glutamate compound to specifically apply glutamate to various types of dendritic spine in rat CA1 pyramidal neurons, thereby allowing selective induction of NMDAR-dependent long-term plasticity. They coupled this method with $\mathrm{Ca}^{2+}$ imaging to determine the relationship between the structure of spines in these neurons and NMDAR-mediated $\mathrm{Ca}^{2+}$ signalling.

First, the authors showed that the extent of NMDAR-mediated current in spines, and therefore, by implica- tion, the expression of functional NMDARs, was positively correlated with the volume of spine heads. Next, they investigated the effects of spine neck structure on $\mathrm{Ca}^{2+}$ signalling. They showed that small spines tend to have narrow necks, which hinders the flow of $\mathrm{Ca}^{2+}$ into the dendritic shaft, and, consequently, there is a larger increase in $\left[\mathrm{Ca}^{2+}\right]_{i}$ in the spine compartments relative to concentrations in spines with larger necks.

Although more work is needed to refine our understanding of spine structure, these findings provide a new link between spine structure and synaptic plasticity, and reveal that the spine head and neck have distinct roles in the regulation of NMDAR-mediated $\mathrm{Ca}^{2+}$ signalling. The volume of the spine head is important for the regulation of synaptic transmission, possibly through variations in expression levels of functional glutamate receptors. By contrast, the specific structure of the spine neck seems to directly influence synaptic $\mathrm{Ca}^{2+}$ signalling in dendrites, and is therefore a determining factor in the selective induction of synaptic plasticity.

Alison Rowan

Q) References and links ORIGINAL RESEARCH PAPER Noguchi, J. et al. Spine-neck geometry determines NMDA receptordependent $\mathrm{Ca}^{2+}$ signaling in dendrites. Neuron 46 609-622 (2005)

FURTHER READING Segal, M. Dendritic spines and long-term plasticity. Nature Rev. Neurosci. 6 , 277-284 (2005)

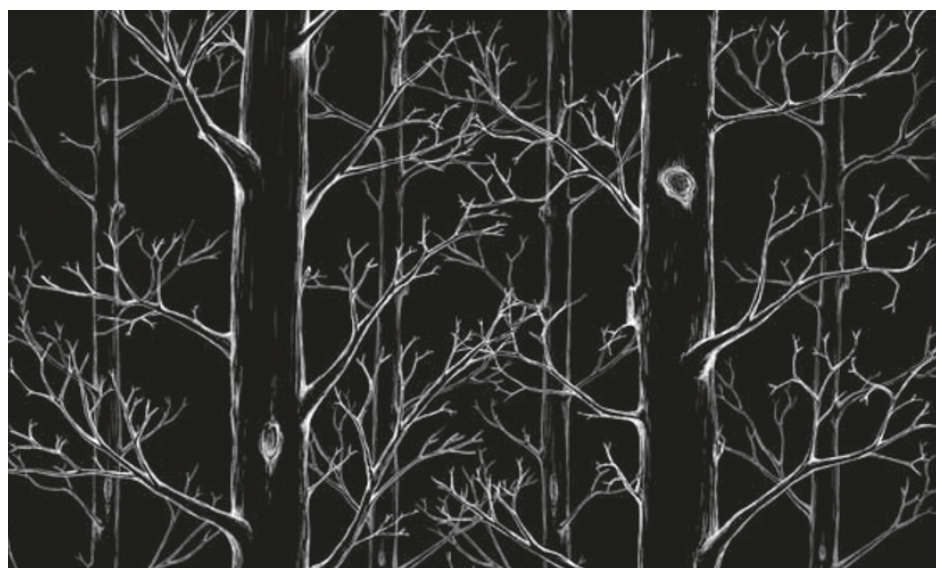

\section{IN BRIEF}

\section{HIPPOCAMPUS}

Attractor dynamics in the hippocampal representation of the local environment.

Wills, T. J. et al. Science $\mathbf{3 0 8}, 873-876$ (2005)

Place cells in the hippocampus respond to specific locations within an environment, and can 'remap' if an animal is moved to a different environment. Wills and colleagues allowed rats to forage in square and circular boxes made of different materials, so that their place cells would form representations of the two environments, and found that the place cell activity resembled attractor states: when rats were subsequently placed in boxes of varying shapes, the activity patterns switched abruptly and simultaneously from one representation to the other.

\section{SYNAPTIC PHYSIOLOGY}

Fast vesicle replenishment allows indefatigable signalling at the first auditory synapse.

Griesinger, C. B. et al. Nature 435, 212-215 (2005)

Although the readily releasable pool of docked vesicles in the ribbon synapses of mammalian inner hair cells contains about the same number of vesicles as those of other synapses, ribbon synapses release vesicles more quickly and with greater temporal precision. Griesinger et al. used two-photon imaging of release sites in the cochlea to show that compartments in the cytoplasm of inner hair cells generate preformed vesicles, and that these vesicles are used to rapidly replenish the readily releasable pool.

\section{LEARNING AND MEMORY}

Disrupting the melanin-concentrating hormone receptor 1 in mice leads to cognitive deficits and alterations of NMDA receptor function.

Adamantidis, A. et al. Eur. J. Neurosci. 21, 2837-2844 (2005)

Mice that lack the gene for the melanin-concentrating hormone $(\mathrm{MCH})$ receptor 1 show a specific deficit on an inhibitory passive avoidance test, and reduced responses to NMDA ( $N$-methyl-Daspartate) in CA1 hippocampal pyramidal neurons. Expression of the NMDA receptor 1 (NMDAR1) subunit in this region was reduced, which indicates that $\mathrm{MCH}$ might be involved in modulating the function or expression of NMDARs.

\section{GENES AND BEHAVIOUR}

Lhx6 delineates a pathway mediating innate reproductive behaviors from the amygdala to the hypothalamus.

Choi, G. B. et al. Neuron 46, 647-660 (2005)

Innate reproductive or defensive behaviours are elicited in mammals by conspecific or predator signals, and are controlled by pathways from the amygdala to the hypothalamus. Choi et al. show that the pathway that mediates reproductive behaviour expresses the transcription factor LHX6, whereas the defensive pathway expresses other LHX proteins. The two pathways are anatomically segregated but converge in the ventromedial hypothalamus, and their opposite neurotransmitter phenotypes might allow a 'gate control' mechanism to integrate conflicting cues. 